\title{
Impact of Interventions on Influenza A(H7N9) Virus Activity in Live Poultry Markets
}

\author{
Eric H.Y. Lau*1, Jun Yuan², Kuibiao Li², Connie Leung ${ }^{3}$, Zhicong Yang ${ }^{2}$, Caojun $\mathrm{Xie}^{2}$, \\ Yufei Liư ${ }^{2}$, Biao $\mathrm{Di}^{2}$, Benjamin Cowling ${ }^{1}$, Xiaoping Tang ${ }^{4}$, Gabriel Leung ${ }^{1}$, Malik Peiris ${ }^{1}$ \\ and Ming Wang ${ }^{2}$
}

${ }^{1}$ School of Public Health, University of Hong Kong, Hong Kong, China; ${ }^{2}$ Guangzhou Center for Disease Control and Prevention, Guangzhou, China; ${ }^{3}$ Laboratory Animal Unit, University of Hong Kong, Hong Kong, China; ${ }^{4}$ The Eight People's Hospital of Guangzhou, Guangzhou, China

\section{Objective}

This study assessed the effect of disinfection and closing live poultry markets in China on avian influenza $\mathrm{A}(\mathrm{H} 7 \mathrm{~N} 9)$ virus detection and viability in a natural setting. We characterized virus detection at different sampling sites to assess exposure risk to the general public and live poultry traders.

\section{Introduction}

H7N9 virus emerged in Eastern China in March 2013, which led to $>550$ human cases and $>200$ deaths in 2 years. Live poultry markets (LPMs) are considered as a major source of human H7N9 infections. In late 2013, the virus had spread to the southern provinces including Guangdong. Its provincial capital Guangzhou, detected its first local H7N9 human case in mid-January 2014 and reaching 10 cases in a month. As a response, Guangzhou government announced a two-week city-wide market closure, banning trading and storing of live poultry. Guangzhou Center for Disease Control and Prevention took this opportunity to establish enhanced surveillance on top of the existing routine LPM surveillance, to assess the impact of such on H7N9 viral isolation and survival.

\section{Methods}

We analyzed environmental samples in routine surveillance from 4 retail LPMs collected on the same day immediately before and after disinfection. To further assess the impact of market closure and disinfection on virus activity, enhanced surveillance with 12 rounds of intensive sampling were carried out before, during and after the 2-week city-wide LPM closure, in 3 retail and 1 wholesale LPMs. Samples were collected from different sites, including poultry cages, drinking water for chicken, inner surface of defeathering machines, barrels holding poultry meat, chopping boards, surfaces of processing tables and wastewater. Samples were tested for AIVs by rRT-PCR and also H7N9 by culture.

\section{Results}

Based on the 214 environmental samples collected from the routine surveillance, the pooled estimated reduction ratios were $58.0 \%(95 \%$ CI $8.9 \%-80.6 \%)$ and $64.2 \%(95 \%$ CI $30.6 \%-81.5 \%)$ for H7N9 and AIV respectively after disinfection. A total of 1466 environmental samples were collected from the targeted enhanced LPM sites. The figure shows the AIV and H7N9 detection and isolation rates before, during and after the market closure (period shaded in gray). We also found a higher H7N9 virus detection in chopping boards in retail LPMs and wastewater in wholesale LPMs. During the market closure, H7N9 viral RNA detection and isolation rates in retail markets decreased by $79 \%(95 \%$ CI, $64 \%-88 \%)$ and $92 \%(95 \%$ CI, $58 \%-98 \%$ ), respectively. Viable H7N9 virus could be cultured from wastewater samples collected up to 2 days after market closure. Our findings indicate that poultry workers and the general population are constantly exposed to H7N9 virus at these markets and that market closure and disinfection rapidly reduces the amount of viable virus.

\section{Conclusions}

Market closure and disinfection reduced H7N9 viral RNA contamination in the LPM environment by $>70 \%$ and infectious virus by $>90 \%$. However, live virus could be detected for around 2 days after the intervention, especially in wastewater sources. To strike the balance between minimizing human infection risk, demands of live poultry from the general public and interest of the poultry industry, coordination between the public health and veterinary sector should be strengthened under a "one-health" approach.
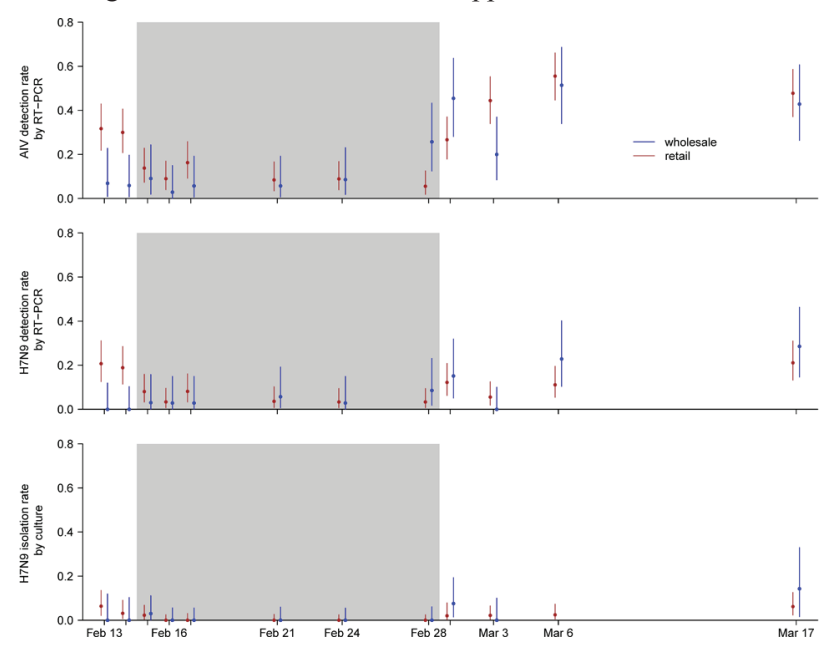

\section{Keywords}

avian influenza; influenza $\mathrm{A}(\mathrm{H} 7 \mathrm{~N} 9)$; live poultry markets; surveillance; disinfection

\section{Acknowledgments}

This work is supported by the National Science and Technology Major Projects of China, Science and Technology Planning Project of Guangdong Province, China, Science and Technology Program of Guangzhou, China, National Natural Science Foundation of China, the Harvard Center for Communicable Disease Dynamics from the National Institute of General Medical Sciences, the Area of Excellence Scheme of the Hong Kong University Grants Committee, and the Health and Medical Research Fund of the Food and Health Bureau, Government of the Hong Kong Special Administrative Region.

\section{*Eric H.Y. Lau}

E-mail: ehylau@hku.hk 